\title{
A Formula of Packing Pressure of a Factor Map
}

\author{
Cao Zhao, Ercai Chen, Xiucheng Hong and Xiaoyao Zhou * \\ School of Mathematical Sciences and Institute of Mathematics, Nanjing Normal University, Nanjing 210023, \\ China; izhaocao@126.com (C.Z.); ecchen@njnu.edu.cn (E.C.); xiuchenghong@163.com (X.H.) \\ * Correspondence: zhouxiaoyaodeyouxian@126.com; Tel.: +153-8081-8424
}

Received: 26 July 2017; Accepted: 30 September 2017; Published: 4 October 2017

Abstract: In this paper, using the notion of packing pressure, we show a formula of packing pressure of a factor map. We also give an application in conformal repellers.

Keywords: packing dimension; packing pressure; factor map

\section{Introduction}

Referring to $(X, T)$ as a topological dynamical system (TDS for short) means that $X$ is a compact metric space and $T$ is a continuous self-map. Entropy is an important topological conjugate invariant in dynamical systems. For a factor map $\pi:(X, T) \rightarrow(Y, S)$, it is obvious that the entropy of $(X, T)$ is larger than the entropy of $(Y, S)$. In general, the converse is false. For example, $(Y, S)$ is a single point system, and $(X, T)$ have positive topological entropy, then $h_{\text {top }}(T)>h_{\text {top }}(S)$. A natural question arises whether there exists the converse inequality. In 1971, Bowen [1] considered a factor map $\pi:(X, T) \rightarrow(Y, S)$, and showed that

$$
h_{\mathrm{top}}(T) \leq h_{\mathrm{top}}(S)+\sup _{y \in Y} h\left(T, \pi^{-1} y\right),
$$

where $h(T, K)$ denotes the entropy of a compact subset $K$ of $X$.

In 1973, Ruelle [2] firstly introduced the notion of topological pressure from statistical mechanics to dynamical systems, which is a generalization of entropy. After that, topological pressure becomes one of basic components in thermodynamics and plays an important role in ergodic theory and dynamical systems.

In 1973, Bowen [3] introduced the notion of topological entropy $h_{\text {top }}^{B}(T, K)$ for arbitrary subset $K$ in a TDS $(X, T)$ in a way resembling Hausdorff dimension. Later, Pesin and Pitskel [4] extended it to topological pressure of any subset.

In 2012, Fang, Huang, Yi and Zhang [5] proved the following entropy formula for a factor map $\pi:(X, T) \rightarrow(Y, S):$

$$
h_{\mathrm{top}}^{B}(T, E) \leq h_{\mathrm{top}}^{B}(S, \pi(E))+\sup _{y \in Y} h\left(T, \pi^{-1}(y)\right)
$$

for any $E \subset X$. Oprocha and Zhang [6] also proved the above formula using open covers. Li , Chen and Zhou [7] extended it to topological pressure.

In dimension theory, packing dimension is considered as important as Hausdorff dimension. The set whose Hausdorff dimension is equal to its packing dimension is called Taylor fractal. It is interesting to consider a set is Taylor fractal or not. Recently, Baek, Olsen, Snigireva [8] and Olsen [9] showed that for many sets of divergence points, the packing dimension and the Hausdorff dimension do not coincide.

Inspired by the approach of defining of the topological entropy of non-compact subset, Feng and Huang [10] introduced the notion of packing entropy in dynamical systems, which resembles 
packing dimension. An understanding of both the topological entropy and the packing entropy of a set provides the basis for substantially better understanding of the underlying dynamical behavior of the set. Similarly, we call the set "dynamical" Taylor fractal if its topological entropy is equal to its packing entropy. In [11], Zhou, Chen and Cheng showed that there are many sets of divergence points which are not "dynamical" Taylor fractals.

Pesin [12] introduced the upper capacity pressure of a set and defined the Caratheodory dimension of a set. The connection between topological pressure and Hausdorff dimension was given by Bowen, who showed that for certain compact sets (quasi-circles) $J \subset \mathbf{C}$ which arise as invariant sets of fractional linear transformations $f$ of the Riemann sphere, the Hausdorff dimension

$$
t^{*}=\operatorname{dim}_{H} J
$$

is the unique root of the equation $P_{J}(-t \varphi)=0$ where $P_{J}$ is the topological pressure of the map $f: J \rightarrow J$, and $\varphi$ is the geometric potential $\varphi(z)=\log \left|f^{\prime}(z)\right|$. Barreira and Schmeling [13] have showed that BS dimension is the unique root of topological pressure function.

Inspired by Pesin [12] and Feng and Huang [10], Wang and Chen [14] generalized it to packing topological pressure. In [14], Wang and Chen also introduced packing version of BS dimension and called it BSP dimension. They also showed BSP dimension is the unique root of packing topological pressure function. Recently, Shi [15] obtained Bowen's equation which establishes the relationship of the packing dimension and packing pressure in dynamical systems with some conditions. This illustrates that packing pressure provides a new technique for the study of dimension theory.

In this paper, we will firstly give a formula of the upper capacity pressure for a factor map. Then we show there is a similar relation of packing topological pressure for a factor map. As an application, we obtain that for a factor map with being finite to one or countable to one, the packing dimension is preservable under the factor map.

\section{Preliminaries and Main Result}

Let $(X, T)$ be a TDS, and $C(X)$ be the set of all continuous functions on $X$. For any $\varphi \in C(X)$, we set $S_{n} \varphi(x):=\sum_{i=0}^{n-1} \varphi\left(T^{i}(x)\right)$. From [16], we can define the following Bowen metric on $X$,

$$
d_{n}(x, y):=\max _{0 \leq i \leq n-1} d\left(T^{i}(x), T^{i}(y)\right), \text { for } x, y, \in X \text { and } n \in \mathbb{N} .
$$

Let $K \subset X$. A subset $E$ is said to be an $(n, \epsilon)$-separated set of $K$, if $x \neq y \in E$ implies that $d_{n}(x, y) \geq \epsilon$. A subset $F$ is said to be an $(n, \epsilon)$-spanning set of $K$, if for any $x \in K$, there exists $y \in F$ such that $d_{n}(x, y)<\epsilon$.

Now, we give a kind of definition of topological pressure resembling Box dimension which is called upper capacity topological pressure.

Definition 1. Reference [12] For any $\varphi \in C(X)$, and any subset $K \subset X$,

$$
P_{n}(\varphi, K, \epsilon)=\inf \left\{\sum_{x \in E} e^{S_{n} \varphi(x)}: E \text { is an }(n, \epsilon) \text {-spanning set of } K\right\} .
$$

The upper capacity topological pressure of $\mathrm{K}$ is given by

$$
P(T, \varphi, K)=\lim _{\epsilon \rightarrow 0} \limsup _{n \rightarrow \infty} \frac{1}{n} \log P_{n}(\varphi, K, \epsilon) .
$$

We can also define the upper capacity topological pressure by separated set. The two definitions are equivalent by a standard proof. Now we state the definition as follows without the proof of the equivalence. 
Definition 2. Reference [12] For any $\varphi \in C(X)$, and any subset $K \subset X$,

$$
Q_{n}(\varphi, K, \epsilon)=\sup \left\{\sum_{x \in F} e^{S_{n} \varphi(x)}: F \text { is an }(n, \epsilon) \text {-separated set of } K\right\} .
$$

The upper capacity topological pressure of $K$ is defined by

$$
P(T, \varphi, K)=\lim _{\epsilon \rightarrow 0} \limsup _{n \rightarrow \infty} \frac{1}{n} \log Q_{n}(\varphi, K, \epsilon) .
$$

Remark that if $\varphi=0$, it is called the upper capacity topological entropy of $K$ and written as $h(T, K)$. In the following, we give the definition of packing dimension before the definition of packing topological pressure.

Definition 3. If $s \geq 0$, and $\delta>0$, let

$$
\mathcal{P}_{\delta}^{\alpha}(Z)=\sup \left\{\sum\left|B_{i}\right|^{\alpha}:\left\{B_{i}\right\} \text { are disjoint closed balls with centers in } Z \text { and }\left|B_{i}\right|<\delta\right\},
$$

where $|A|$ denotes the diameter of $A$. Since $\mathcal{P}_{\delta}^{\alpha}(Z)$ decreases when $\delta$ decreases, $\mathcal{P}_{0}^{\alpha}(Z)=\lim _{\delta \rightarrow 0} \mathcal{P}_{\delta}^{\alpha}$ exists. The $\alpha$-packing measure is defined by

$$
\mathcal{P}^{\alpha}(Z)=\inf \left\{\sum_{i} \mathcal{P}_{0}^{\alpha}\left(Z_{i}\right): Z \subset \bigcup_{i=1}^{\infty} Z_{i}\right\} .
$$

The packing dimension of $\mathrm{Z}$ is defined by

$$
\operatorname{dim}_{P}(Z)=\sup \left\{\alpha: \mathcal{P}^{\alpha}(Z)=\infty\right\}
$$

Now, we give the packing topological pressure on arbitrary set, which is similar to packing dimension.

Definition 4. For a set $Z \subset X$ and $\alpha \in \mathbb{R}, 0<\delta \in \mathbb{R}, \varphi \in C(X)$ and $N \in \mathbb{N}$, let

$$
M(Z, \alpha, \varphi, \delta, N)=\inf _{\mathcal{P}^{*}(Z, N, \epsilon)} \sum_{i=1}^{\infty} \exp \left(-\alpha n_{i}+\sup _{x \in \overline{B_{n_{i}}}\left(x_{i}, \delta\right)} S_{n_{i}} \varphi(x)\right),
$$

where $\mathcal{P}^{*}(Z, N, \epsilon)$ denotes the finite or countable disjoint set family $\left\{B_{n_{i}}\left(x_{i}, \delta\right)\right\}$ with $x_{i} \in Z, n_{i} \geq N$. Clearly, $M(Z, \alpha, \varphi, \delta, N)$ is monotone increasing function about $N$. let

$$
m^{*}(Z, \alpha, \varphi, \delta)=\lim _{N \rightarrow \infty} M(Z, \alpha, \varphi, \delta, N) .
$$

Moreover, let

$$
m^{* *}(Z, \alpha, \varphi, \delta)=\inf \left\{\sum_{i=1}^{\infty} m^{*}\left(Z_{i}, \alpha, \varphi, \delta\right): \bigcup_{i=1}^{\infty} Z_{i} \supset Z\right\}
$$

Then

$$
P^{\text {pack }}(T, \varphi, Z, \delta)=\inf \left\{\alpha: m^{* *}(Z, \alpha, \varphi, \delta)=0\right\}=\sup \left\{\alpha: m^{* *}(Z, \alpha, \varphi, \delta)=+\infty\right\} .
$$

Finally, we define the packing topological pressure $P^{\text {pack }}(T, \varphi, Z):=\lim _{\delta \rightarrow 0} P^{p a c k}(T, \varphi, Z, \delta)$. The packing entropy of $Z$ is written as $h^{\text {pack }}(T, Z)$ as $\varphi=0$.

Let $(X, T)$ and $(Y, S)$ be two TDSs with $(X, d)$ and $(Y, \rho)$ be compact metric spaces, respectively. A continuous surjective map $\pi: X \rightarrow Y$ satisfying $\pi \circ T=S \circ \pi$ means that $(Y, S)$ is a factor of $(X, T)$. Now, we state our main result as follows. 
Theorem 1. Let $\pi:(X, T) \rightarrow(Y, S)$ be a factor map and $\varphi \in C(Y)$. Then for any set $E \subset X$,

(1) $P^{\text {pack }}(S, \varphi, \pi(E)) \leq P^{\text {pack }}(T, \varphi \circ \pi, E) \leq h^{\text {pack }}(S, \pi(E))+\sup _{y \in Y} P\left(T, \varphi \circ \pi, \pi^{-1}\{y\}\right)$.

(2) $P^{\text {pack }}(S, \varphi, \pi(E)) \leq P^{\text {pack }}(T, \varphi \circ \pi, E) \leq P^{\text {pack }}(S, \varphi, \pi(E))+\sup _{y \in Y} h\left(T, \pi^{-1}\{y\}\right)$.

\section{Proof of Theorem 1}

In this section, we are going to prove the main result of this paper. In the following, for any $c>0$ and $\varphi \in C(Y)$, we set

$$
\operatorname{Var}(\varphi, c)=\sup _{x, y \in Y} \mid\{\varphi(x)-\varphi(y) \mid: \rho(x, y) \leq c\} .
$$

To prove the main result, we need the following lemma. The original idea follows from Bowen [1], which compared the entropy of two topological semi-conjugacy systems. Later, Fang, Huang, Yi and Zhang [5] and Oprocha and Zhang [6] extended it to comparing the Bowen entropy of the subset under a factor map. And Li, Chen and Zhou [7] generalized it to Pesin topological pressure. The lemma here is devoted to the study of the upper capacity pressure. Since the proof is standard and similar to the above references, we only present the sketch of the proof.

Lemma 1. Let $\pi:(X, T) \rightarrow(Y, S)$ be a factor map and $\varphi \in C(Y)$. Then for any set $E \subset X$,

(1) $P(S, \varphi, \pi(E)) \leq P(T, \varphi \circ \pi, E) \leq h(S, \pi(E))+\sup _{y \in Y} P\left(T, \varphi \circ \pi, \pi^{-1}\{y\}\right)$.

(2) $P(S, \varphi, \pi(E)) \leq P(T, \varphi \circ \pi, E) \leq P(S, \varphi, \pi(E))+\sup _{y \in Y} h\left(T, \pi^{-1}\{y\}\right)$.

Proof. (1) Clearly, we have $P(S, \varphi, \pi(E)) \leq P(T, \varphi \circ \pi, E)$. We only need prove the second inequality. Assume that $b:=\sup _{y \in Y} P\left(T, \varphi \circ \pi, \pi^{-1}(y)\right)<\infty$, since $b=\infty$, the proof is clear. Fix $\epsilon>0$ and $\tau>0$. For any $y \in Y$ choose $m(y) \in \mathbb{N}$ large enough such that there exists an $(m(y), \epsilon)$-spanning set $E_{y}$ of $\pi^{-1}(y)$ and

$$
\sum_{x \in E_{y}} \exp \left(S_{m(y)} \varphi \circ \pi(x)\right) \leq \exp ((b+\tau) m(y)) .
$$

Following from the proof of Theorem 2.1(ii) in [7], and choosing $\delta>0$ small enough, for any $y \in Y$, we can choose $t_{0}, \cdots, t_{q}$ and $\Delta_{y}\left(t_{0}(y)\right), \Delta_{y}\left(t_{1}(y)\right) \cdots, \Delta_{y}\left(t_{q(y)}(y)\right) \in Y$ depending on $y$ recursively such that $t_{0}(y)=0$ and $t_{s+1}(y)=t_{s}(y)+m\left(\Delta_{y}\left(t_{s}(y)\right)\right)$ until $t_{q+1}(y) \geq n$. For any $z_{0} \in E_{\Delta_{y}\left(t_{0}(y)\right)}, z_{1} \in$ $E_{\Delta_{y}\left(t_{1}(y)\right)} \cdots, z_{q(y)} \in E_{\Delta_{y}\left(t_{q(y)}(y)\right)}$ we consider the following set

$V\left(y ; z_{0}, \cdots, z_{q(y)}\right)=\left\{x \in X: d\left(T^{t+t_{s}(y)}(x), T^{t}\left(x_{s}\right)\right)<2 \epsilon\right.$ for all $0 \leq t<m\left(\Delta_{t_{s}}(y)\right)$ and $\left.0 \leq s \leq q(y)\right\}$.

Then we have

- $\left\{V\left(y ; z_{0}, \cdots, z_{q(y)}\right): z_{0} \in E_{\Delta_{y}\left(t_{0}(y)\right)}, z_{1} \in E_{\Delta_{y}\left(t_{1}(y)\right)} \cdots, z_{q(y)} \in E_{\Delta_{y}\left(t_{q(y)}(y)\right)}\right\} \supset \pi^{-1}\left(B_{n}(y, \delta)\right)$.

- $\quad \sum_{z_{0} \in E_{\Delta y}\left(t_{0}(y)\right), z_{1} \in E_{\Delta y}\left(t_{1}(y)\right)} \cdots, z_{q(y)} \in E_{\Delta y\left(t t_{(y)}(y)\right)} \exp \left(S_{n} \varphi \circ \pi\left(v\left(z_{0}, \cdots, z_{q(y)}\right)\right)\right) \leq \exp ((b+\tau+\operatorname{Var}(\varphi \circ$ $\pi, 2 \epsilon)) n) \exp ((|b|+\tau) M+M\|\varphi\|)$.

For any $n \in \mathbb{N}$ and sufficiently small $\delta>0$, we let $W_{n}$ be an $(n, \delta)$ spanning set of $\pi(E)$ with

$$
\# W_{n} \leq \exp (n(h(S, \pi(E))+\tau)) .
$$


And $H_{n}:=\left\{v\left(z_{0}, \cdots, z_{q(w)}\right): w \in W_{n}, z_{0} \in E_{\Delta_{w}\left(t_{0}(w)\right)}, z_{1} \in E_{\Delta_{w}\left(t_{1}(w)\right)} \cdots, z_{q(w)} \in E_{\Delta_{w}\left(t_{q(w)}(w)\right)}\right\}$ is an $(n, 4 \epsilon)$-spanning set of $E$. Since $w \in W_{n}$, we have

$$
\begin{aligned}
& \sum_{z_{0} \in E_{\Delta_{w}\left(t_{0}(w)\right)}, z_{1} \in E_{\Delta_{w}\left(t_{1}(w)\right)} \cdots, z_{q(w)} \in E_{\Delta_{w}\left(t_{q(w)}(w)\right)}} \exp \left(S_{n} \varphi \circ \pi\left(v\left(z_{0}, \cdots, z_{q(w)}\right)\right)\right) \\
& \leq \exp ((b+\tau+\operatorname{Var}(\varphi \circ \pi, 2 \epsilon)) n) \exp ((|b|+\tau) M+M\|\varphi\|) .
\end{aligned}
$$

Combing with Equation (1), we have

$$
\begin{aligned}
P_{n}(\varphi \circ \pi, E, 4 \epsilon) & \leq \sum_{v \in H_{n}} \exp \left(S_{n} \varphi \circ \pi(v)\right) \\
& \leq \# W_{n} \exp ((b+\tau+\operatorname{Var}(\varphi \circ \pi, 2 \epsilon)) n) \exp ((|b|+\tau) M+M\|\varphi\|) .
\end{aligned}
$$

Moreover,

$$
\frac{\log P_{n}(\varphi \circ \pi, E, 4 \epsilon)}{n} \leq h(S, \pi(E))+\tau+b+\tau+\operatorname{Var}(\varphi \circ \pi, 2 \epsilon)+\frac{(|b|+\tau) M+M\|\varphi\|}{n} .
$$

Letting $n \rightarrow \infty, \epsilon \rightarrow 0$ and $\tau \rightarrow 0$, we finish the proof.

(2) We can assume that $a=\sup _{y \in Y} h\left(T, \pi^{-1}(y)\right)<\infty$, since if $a=\infty$, the proof is finished. Fix $\epsilon>0$ and $\tau>0$. Use the same techniques as the proof in [5] (Theorem 3.3) or [7] (Theorem 2.1 (i)) there exists $l(y)>0$ and $v_{1}(y), v_{2}(y), \cdots, v_{l(y)}(y) \in X$ such that

- $l(y) \leq \exp ((a+\tau)(n+M))$;

- $\quad \cup_{i=1}^{l(y)} B_{n}\left(v_{i}(y), 4 \epsilon\right) \supset \pi^{-1} B_{n}(y, \delta)$;

- $\pi B_{n}\left(v_{i}(y), 4 \epsilon\right) \cap B_{n}(y, \delta) \neq \varnothing$ for any $1 \leq i \leq l(y)$.

For any $n \in \mathbb{N}$ and sufficiently small $\delta>0$, let $W_{n}$ be an $(n, \delta)$ spanning set of $\pi(E)$ with

$$
\sum_{w \in W_{n}} \exp \left(S_{n} \varphi(x)\right) \leq \exp (n(P(S, \varphi, \pi(E))+\tau)) .
$$

For each $w \in W_{n}, B_{n}(w, \delta)$, we have

- $\quad l(w) \leq \exp ((a+\tau)(n+M))$;

- $\quad \cup_{i=1}^{l(w)} B_{n}\left(v_{i}(w), 4 \epsilon\right) \supset \pi^{-1} B_{n}(w, \delta)$

- $\pi B_{n}\left(v_{i}(w), 4 \epsilon\right) \cap B_{n}(w, \delta) \neq \varnothing$ for any $1 \leq i \leq l(w)$.

Furthermore,

$$
\cup_{w \in W_{n}} \cup_{i=1}^{l(w)} B_{n}\left(v_{i}(w), 4 \epsilon\right) \supset \cup_{w \in W_{n}} \pi^{-1} B_{n}(w, \delta) \supset \pi^{-1}(\pi(E)) \supset E .
$$

This means that $H_{n}^{\prime}:=\left\{v_{i}(w), w \in W_{n}, 1 \leq i \leq l(w)\right\}$ is an $(n, 4 \epsilon)$-spanning set of $E$. Then we have

$$
\begin{aligned}
P_{n}(\varphi \circ \pi, E, 4 \epsilon) & \leq \sum_{v \in H_{n}^{\prime}} \exp \left(S_{n} \varphi \circ \pi(v)\right) \\
& \left.\leq \sum_{w \in W_{n}} \sum_{i=1}^{l(w)} \exp S_{n} \varphi \circ \pi\left(v_{i}(w)\right)\right) \\
& \leq \sum_{w \in W_{n}} l(w) \exp \left(S_{n} \varphi(w)+n \operatorname{Var}(\varphi, \delta)+n \operatorname{Var}(\varphi \circ \pi, 4 \epsilon)\right) \\
& \leq \exp ((a+\tau) n) \sum_{w \in W_{n}} \exp \left(S_{n} \varphi(w)\right)
\end{aligned}
$$


Following from Equation (2), we have

$$
\frac{\log P_{n}(\varphi \circ \pi, E, 4 \epsilon)}{n} \leq a+\tau+P(S, \varphi, \pi(E))+\tau .
$$

Letting $\epsilon \rightarrow, \tau \rightarrow 0$, we finish the proof.

We introduce the following lemma, which is similar to the packing dimension.

Lemma 2. For any subset $Z \subset X$, we have

$$
P^{p a c k}(T, \varphi, Z)=\inf \left\{\sup _{i} P\left(T, \varphi, Z_{i}\right): \cup_{i=1}^{\infty} Z_{i}=Z\right\} .
$$

Proof. Fix any $a>P^{\text {pack }}(T, \varphi, Z)=\lim _{\delta \rightarrow 0} P^{\text {pack }}(T, \varphi, Z, \delta)$. There exists some $\delta>0$ such that $P^{\text {pack }}(T, \varphi, Z, \delta)<a$, so $m^{* *}(Z, a, \varphi, \delta)=0$. We can choose $\left\{Z_{i}\right\}_{i=1}^{\infty}$ such that $\bigcup_{i=1}^{\infty} Z_{i} \supset Z$ and $\sum_{i=1}^{\infty} m^{*}\left(Z_{i}, a, \varphi, \delta\right)<1$. So for each $i, N$ large enough, we have $M\left(Z_{i}, a, \varphi, \delta, N\right)<1$. Next, we will prove for any $i$,

$$
P\left(T, \varphi, Z_{i}\right) \leq a .
$$

For any $(N, 3 \delta)$ separated set $E_{i}$ of $Z_{i}$, we can choose disjoint set family $\left\{B_{N}\left(x_{i}, \delta\right)\right\}$ with $x_{i} \in$ $E_{i} \subset Z_{i}$. Furthermore,

$$
\sum_{x \in E_{i}} e^{-N a+S_{N} \varphi(x)} \leq M\left(Z_{i}, a, \varphi, \delta, N\right)<1 .
$$

Then

$$
\sum_{x \in E_{i}} e^{S_{N} \varphi(x)} \leq e^{N a}
$$

Moreover,

$$
P\left(T, \varphi, Z_{i}, 3 \delta\right) \leq a+\operatorname{Var}(\varphi, 3 \delta)
$$

Let $\delta \rightarrow 0$, we finish the proof.

Now we show the converse inequality. Fix any $0<t<s<P^{\text {pack }}(T, \varphi, Z)$ and any $\cup_{i=1}^{\infty} Z_{i}=Z$, we only need to prove that there exists some $i \geq 1$ such that $P\left(T, \varphi, Z_{i}\right)>t$. Since $s<P^{\text {pack }}(T, \varphi, Z)$, we can choose $\delta>0$ such that $m^{* *}(Z, s, \varphi, \delta)>0$, moreover, there exists $\alpha>0$ and some $Z_{i}$ such that $m^{*}\left(Z_{i}, s, \varphi, \delta\right)>\alpha$. Furthermore, we can choose $N>0$ and a family of disjoint set family $\left\{B_{n_{j}}\left(x_{j}, \delta\right)\right\}$ with $x_{j} \in Z_{i}, j \geq 1, n_{j} \geq N$ such that

$$
\sum_{j=1}^{\infty} \exp \left(-s n_{j}+\sup _{x \in \bar{B}_{n_{j}}\left(x_{j}, \delta\right)} S_{n_{j}} \varphi(x)\right) \geq \frac{\alpha}{2} .
$$

Let $E_{k}=\left\{x_{j}: n_{j}=k\right\}$, then

$$
\sum_{k} \sum_{x \in E_{k}} \exp \left(-s k+\sup _{x \in \overline{B_{k}}(x, \delta)} S_{k} \varphi(x)\right) \geq \frac{\alpha}{2}
$$

There exists $k \geq N$ such that

$$
\sum_{x \in E_{k}} \exp \left(\sup _{x \in \overline{B_{k}(x, \delta)}} S_{k} \varphi(x)\right) \geq \frac{e^{k t}\left(1-e^{t-s}\right) \alpha}{2} .
$$

since otherwise

$$
\sum_{k} \sum_{x \in E_{k}} \exp \left(-s k+\sup _{x \in \overline{B_{k}}(x, \delta)} S_{k} \varphi(x)\right) \leq \sum_{k} \frac{e^{-s k} e^{k t}\left(1-e^{t-s}\right) \alpha}{2} \leq \frac{\alpha}{2} .
$$


Hence, $E_{k}$ is an $(k, \epsilon)$ separated set of $Z_{i}$, one obtain

$$
Q_{k}\left(\varphi, Z_{i}, \epsilon\right)=\sup \left\{\sum_{x \in F} e^{S_{n} \varphi(x)}: F \text { is an }(k, \epsilon) \text { separated set of } Z_{i}\right\} \geq \frac{e^{k t}\left(1-e^{t-s}\right) \alpha}{2} .
$$

Then we have $P\left(T, \varphi, Z_{i}\right)>t$.

Proof. Proof of Theorem 1

(1) By Lemma 2,

$$
P^{\text {pack }}(S, \varphi, \pi(E))=\inf \left\{\sup _{i} P\left(S, \varphi, Z_{i}\right): \cup_{i=1}^{\infty} Z_{i}=\pi(E)\right\} .
$$

Consider the set $\pi(E)=\cup_{i=1}^{\infty} Z_{i}$, one obtain $E \subset \pi^{-1}\left(\cup_{i=1}^{\infty} Z_{i}\right)=\cup_{i=1}^{\infty} \pi^{-1}\left(Z_{i}\right)$. So $E=$ $\cup_{i=1}^{\infty} \pi^{-1}\left(Z_{i}\right) \cap E$, hence, we have

$$
\begin{aligned}
P^{\text {pack }}(T, \varphi \circ \pi, E) & \leq \inf \left\{\sup _{i} P\left(T, \varphi \circ \pi, \pi^{-1} Z_{i} \cap E\right): \cup_{i=1}^{\infty} Z_{i}=\pi(E)\right\} \\
& \leq \inf \left\{\sup _{i} P\left(T, \varphi \circ \pi, \pi^{-1} Z_{i}\right): \cup_{i=1}^{\infty} Z_{i}=\pi(E)\right\} .
\end{aligned}
$$

It follows from Lemma 1 that

$$
P\left(T, \varphi \circ \pi, \pi^{-1} Z_{i}\right) \leq P\left(S, \varphi, Z_{i}\right)+\sup _{y \in Y} h\left(T, \pi^{-1}\{y\}\right) .
$$

Then we have

$$
\begin{aligned}
P^{\text {pack }}(T, \varphi \circ \pi, E) & \leq \inf \left\{\sup _{i} P\left(T, \varphi \circ \pi, \pi^{-1} Z_{i}\right): \cup_{i=1}^{\infty} Z_{i}=\pi(E)\right\} \\
& \leq \inf \left\{\sup _{i} P\left(S, \varphi, Z_{i}\right): \cup_{i=1}^{\infty} Z_{i}=\pi(E)\right\}+\sup _{y \in Y} h\left(T, \pi^{-1}\{y\}\right) \\
& =P^{\text {pack }}(S, \varphi, Z)+\sup _{y \in Y} h\left(T, \pi^{-1}\{y\}\right) .
\end{aligned}
$$

(2) Following from Lemma 1, and the above Equation (3), we have

$$
P\left(T, \varphi \circ \pi, \pi^{-1} Z_{i}\right) \leq h\left(S, Z_{i}\right)+\sup _{y \in Y} P\left(T, \varphi \circ \pi, \pi^{-1}\{y\}\right) .
$$

Hence,

$$
\begin{aligned}
P^{\text {pack }}(T, \varphi \circ \pi, E) & \left.\leq \inf _{i} \sup _{i} h\left(S, Z_{i}\right): \cup_{i=1}^{\infty} Z_{i}=\pi(E)\right\}+\sup _{y \in Y} P\left(T, \varphi \circ \pi, \pi^{-1}\{y\}\right) \\
& =h^{\text {pack }}(S, \pi(E))+\sup _{y \in Y} P\left(T, \varphi \circ \pi, \pi^{-1}\{y\}\right) .
\end{aligned}
$$

So we finish the proof.

\section{Applications}

Let $f: M \rightarrow M$ be a $C^{1+\alpha}$ map of smooth manifold, and $J$ an $f$-invariant compact subset of $M$. We say that $f$ is expanding on $J$ and $J$ is repeller of $f$, if there are constants $c>0$ and $\beta=\lambda^{-1}>1$ such that $\left\|d_{x} f^{n} u\right\| \geq c \beta^{n}\|u\|$ for any $x \in M, u \in T_{x} M$ and $n \geq 1$. If in addition the derivative of $f$ is a scalar multiple of an isometry at any point of $J$ we call $J$ a conformal repeller.

In the case of the symbolic dynamical system $\left(\Sigma_{A}^{+}, \sigma\right)$ we can consider a coding map $\chi: \Sigma_{A}^{+} \rightarrow J$ for the repeller, which is Hölder continuous, onto, and satisfies $f \circ \chi=\chi \circ \sigma$ and $\sup \left\{\#\left(\chi^{-1}(x)\right): x \in\right.$ $J\}<\infty$ (see, for example, Reference [17] for details). Therefore, we apply Theorem 1 to obtain the below result. 
Proposition 1. Let $f: J \rightarrow J$ be conformal expanding on $J, \chi: \Sigma_{A}^{+} \rightarrow J$ a coding map and $\varphi: J \rightarrow \mathbb{R}$ be a continuous function. Then for each set $E \subset \Sigma_{A}^{+}$, we have

$$
P^{\text {pack }}(\sigma, \varphi \circ \chi, E)=P^{\text {pack }}(f, \varphi, \chi(E)) .
$$

Following from the definition of the BS packing dimension introduced by Wang and Chen [14] and the Proposition 1, we have the following corollary.

Corollary 1. Let $f: J \rightarrow J$ be conformal expanding on $J, \chi: \Sigma_{A}^{+} \rightarrow J$ a coding map. Then for each set $E \subset \Sigma_{A^{\prime}}^{+}$ we have

$$
\operatorname{dim}_{P} E=\operatorname{dim}_{P}(\chi(E)) .
$$

Example: (Sierpiński triangle) Consider a special IFS (see Figure 1) as:

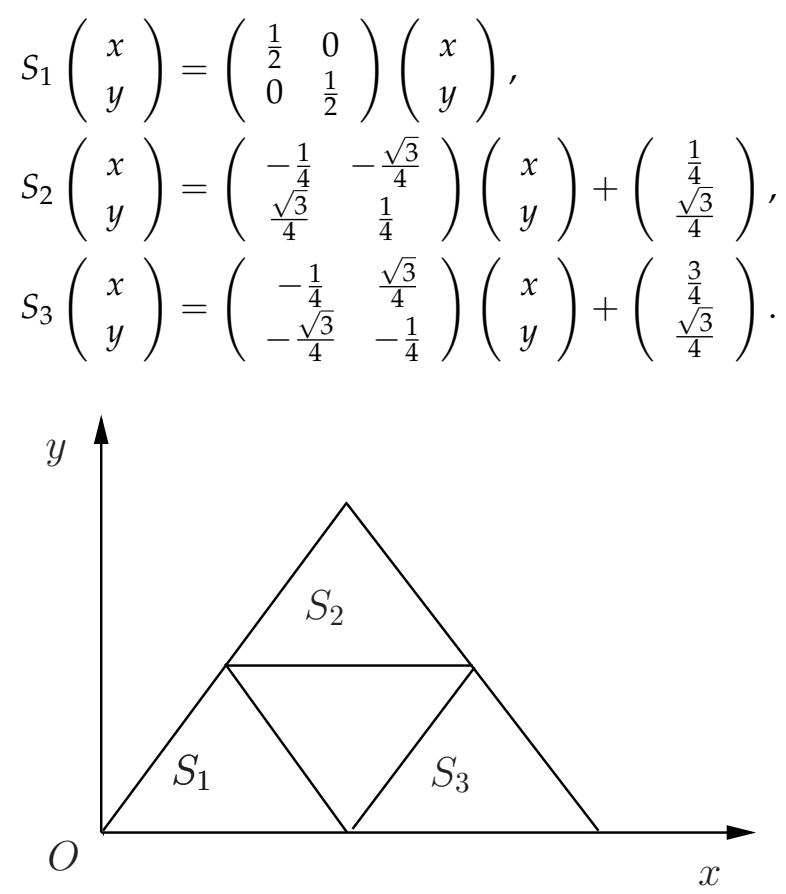

Figure 1. Sierpiński triangle.

Let $E$ be the Sierpiński gasket generated by $S_{i}, i=1,2,3$. We define $F: E \rightarrow E$ by

$$
F(x)=S_{j}^{-1} x, x \in S_{j}(E) .
$$

Let $\Sigma=\{1,2,3\}^{\mathbb{N}}$. Then for any $\omega=\left(\omega_{1}, \omega_{2} \cdots\right) \in \Sigma^{+}$, we can define

$$
\chi(\omega)=\bigcap_{n \geq 1} S_{\omega_{n}} \cdots \circ S_{\omega_{2}} \circ S_{\omega_{1}}(E) .
$$

Then $F: E \rightarrow E$ is conformal expanding on $E, \chi: \Sigma \rightarrow E$ a coding map. Then $(E, F)$ satisfies the condition of Proposition 1. Hence, we have for any $K \subset \Sigma$,

$$
P^{\text {pack }}(\sigma, \varphi \circ \chi, K)=P^{\text {pack }}(F, \varphi, \chi(K)) .
$$

Acknowledgments: The authors thank the anonymous referees for their valuable suggestions to improve the quality of this article. The fourth author was supported by NNSF of China (11601235), NSF of Jiangsu Province 
(BK20161014), NSF of the Jiangsu Higher Education Institutions of China (16KJD110003), China Postdoctoral Science Foundation (2016M591873) and China Postdoctoral Science Special Foundation (2017T100384). The second author was supported by NNSF of China $(11671208,11431012)$. The work was also funded by the Priority Academic Program Development of Jiangsu Higher Education Institutions.

Author Contributions: Ercai Chen and Xiaoyao Zhou conceived the idea; Xiucheng Hong analyzed the data; Cao Zhao wrote the paper.

Conflicts of Interest: The authors declare no conflict of interest.

\section{References}

1. Bowen, R. Entropy for group endomorphisms and homogeneous spaces. Trans. Am. Math. Soc. 1971, 153, 401-414.

2. Ruelle, D. Statistical mechanics on a compact set with $Z^{m}$ action satisfying expansiveness and specification. Trans. Am. Math. Soc. 1973, 185, 79-122.

3. Bowen, R. Topological entropy for non-compact sets. Trans. Am. Math. Soc. 1973, 184, 125-136.

4. Pesin, Y.; Pitskel, B. Topological pressure and the variational principle principle for non-compact sets. Func. Anal. Appl. 1984, 18, 307-318.

5. Fang, C.; Huang, W.; Yi, Y.; Zhang, P. Dimensions of stable sets and scrambled sets in positive finite entropy systems. Ergod. Theory Dyn. Syst. 2012, 32, 599-628.

6. Oprocha, P.; Zhang, G. Dimensional entropy over sets and fibres. Nonlinearity 2011, 24, 2325-2346.

7. Li, Q.; Chen, E.; Zhou, X. Corrigendum to: "A note of topological pressure for non-compact sets of a factor map". Chaos Solitions Fractal 2013, 53, 75-77.

8. Baek, I.; Olsen, L.; Snigireva, N. Divergence points of self-similar measures and packing dimension. Adv. Math. 2007, 214, 267-287.

9. Olsen, L. Multifractal analysis of divergence points of deformed measure theoretical Birkhoff averages. IV. Divergence points and packing dimension. Bull. Sci. Math. 2008, 132, 650-678.

10. Feng, D.; Huang, W. Variational principles for topological entropies of subsets. J. Func. Anal. 2012, 263, 2228-2254.

11. Zhou, X.; Chen, E.; Cheng, W.C. Packing entropy and divergence points. Dyn. Syst. 2012, 27, 387-402.

12. Pesin, Y. Dimension Theory in Dynamical Systems: Contemporary Views and Applications; University of Chicago Press: Chicago, IL, USA, 1997.

13. Barreira, L.; Schmeling, J. Sets of non-typical points have full topological entropy and full Hausdorff dimension. Isr. J. Math. 2000, 116, 29-70.

14. Wang, C.; Chen, E. Variational principles for BS dimension of subsets. Dyn. Syst. 2012, 27, 359-385.

15. Shi, Q. Bowen's Equation of the Packing Dimension in Dynamical Systems. Mater's Dissertation, Research Paper, 2012. (in Chinese)

16. Walters, P. An Introduction to Ergodic Theory; Springer-Verlag: Berlin, Germany, 1982.

17. Schmeling, J. Entropy preservation under Markov coding. J. Stat. Phys. 2001, 104, 799-815. 